\title{
EFFECT OF ANNEALING ON THE AC CONDUCTIVITY AND THE DIELECTRIC PROPERTIES OF $\mathrm{In}_{2} \mathrm{Te}_{3}$ THIN FILMS
}

\author{
M.A. Afifi, E. AbD El-WahabB, A.E. Bekheet and H.E. Atyia \\ Physics Department, Faculty of Education, Ain Shams University, Roxy, Cairo, Egypt \\ (Received April 26, 2000; revised version July 13, 2000)
}

$\mathrm{In}_{2} \mathrm{Te}_{3}$ thin films were prepared by thermal evaporation technique. The composition of the films is checked by energy dispersive X-ray analysis. X-ray analysis showed that the as-deposited $\mathrm{In}_{2} \mathrm{Te}_{3}$ films as well as films annealed at temperatures $\leq 473 \mathrm{~K}$ have crystalline structure. The ac conductivity $\sigma_{\mathrm{ac}}(\omega)$, the dielectric constant $\varepsilon_{1}$ and the dielectric loss $\varepsilon_{2}$ of $\operatorname{In}_{2} \mathrm{Te}_{3}$ films were studied in the temperature range $303-373 \mathrm{~K}$ and in the frequency range $100 \mathrm{~Hz}-100 \mathrm{kHz}$. The ac conduction activation energy $\Delta E_{\sigma}(\omega)$ was found to be $0.065 \mathrm{eV}$ for the as-deposited films. The ac conductivity was found to obey the relation $\sigma_{\mathrm{ac}}(\omega)=A \omega^{s}$, where $s$ is the frequency exponent. The obtained temperature dependence of $s$ is reasonably interpreted by quantum mechanical tunneling model. Both the dielectric constant $\varepsilon_{1}$ and the dielectric loss $\varepsilon_{2}$ increased with temperature and decreased with frequency in the investigated range. The frequency and temperature dependencies of $\sigma_{\mathrm{ac}}(\omega), \varepsilon_{1}$, and $\varepsilon_{2}$ for the annealed samples have the same behavior as that for the as-deposited samples. However, values of $\sigma_{\mathrm{ac}}(\omega), \varepsilon_{1}$, and $\varepsilon_{2}$ measured at any frequency and temperature increased with annealing temperature up to $473 \mathrm{~K}$. It was found also that $\Delta E_{\sigma}(\omega)$ decreased with annealing temperature.

PACS numbers: 72.20.-i, 77.55. $+f$

\section{Introduction}

Although many recent works have dealt with $\mathrm{A}_{2}^{\mathrm{III}} \mathrm{B}_{3}^{\mathrm{VI}}$ compounds with $\mathrm{A}=\mathrm{Ga}$, In, $\mathrm{Tl}$ and $\mathrm{B}=\mathrm{S}, \mathrm{Se}, \mathrm{Te}$, their properties remain rather confusing. These compounds have mainly zinc-blende and wurtzite structures with tetrahedral [1] bonding. They are the simplest types of defective structures in which some of the crystallographically equivalent sites are only partially occupied and that the lattice has vacant sites [2]. The presence of a large number of defects destroys the periodicity of the lattice and distorts the crystal field. Therefore, the semiconducting properties of these materials should be strongly affected by annealing which increases the degree of ordering and hence affects their physical properties. 
$\mathrm{In}_{2} \mathrm{Te}_{3}$ is a member of the above-mentioned compounds, has two phases ( $\alpha$ and $\beta$ ) [3-6]. $\beta$-phase has a zinc-blende structure [7] which is characterized by a completely random distribution of the metal ions in their sublattice. It can be transformed to the ordered state ( $\alpha$-phase) as obtained by Kosevich et al. [8]. Zahab et al. [9] have shown that an appropriate annealing of $\mathrm{In}_{2} \mathrm{Te}_{3}$ films leads to a polycrystalline $\beta$-phase with a preferred orientation in the [111] direction. Several authors investigated the electrical properties of $\mathrm{In}_{2} \mathrm{Te}_{3}$ thin films [9-13]. Papers on the ac conductivity and the dielectric properties of $\operatorname{In}_{2} \mathrm{Te}_{3}$ are rare.

Measurements of ac conductivity of chalcogenide semiconductors have been extensively used to interpret the conduction process in these materials. For the mechanism of the ac conductivity, the quantum mechanical tunneling (QMT) model was proposed by Pollak and Geballe [14] to interpret impurity conduction in $n$-type silicon.

This work is aimed to investigate the temperature and frequency dependence of the ac conductivity and the dielectric properties of $\operatorname{In}_{2} \mathrm{Te}_{3}$ thin films of different thicknesses. Also, the effect of annealing at different temperatures on these properties has been investigated. The results are discussed on the base of QMT theory of ac conductivity.

\section{Experimental technique}

$\mathrm{In}_{2} \mathrm{Te}_{3}$ was synthesized $[13,15]$ by direct fusion of stoichiometric amounts of indium and tellurium (purity $99.999 \%$ ) in an evacuated sealed silica tube $\left(10^{-5}\right.$ Torr). Thin films with different thicknesses of $\mathrm{In}_{2} \mathrm{Te}_{3}$ were obtained by thermal evaporation of the investigated composition onto cleaned glass substrates. $\mathrm{X}$-ray diffraction technique was used to investigate the structure of the investigated films, using Philips PM 8203 diffractometer. The chemical composition of the obtained films was checked by energy dispersive X-ray analysis (EDX) using scanning electron microscope (Joel 5400). For dc and ac measurements, films were sandwiched between two $\mathrm{Al}$ electrodes. The resistance $(R)$ was measured using an electrometer (Keithley model 616 ). The dc conductivity $\sigma_{\mathrm{dc}}$ is related to the electrical resistance by the relation

$$
\sigma_{\mathrm{dc}}=d / R A,
$$

where $d$ is the thickness of the film and $A$ is the cross-sectional area. The dielectric constant $\varepsilon_{1}$, the dielectric loss $\varepsilon_{2}$, and the ac conductivity $\sigma_{\text {ac }}$ were measured using programmable automatic RCL meter (Philips PM 6304) which measures the impedance $Z$, the capacitance $C$ and the loss $\operatorname{tangent}(\tan \delta)$ directly. The dielectric constant $\varepsilon_{1}$ (real part of the dielectric constant) was calculated using the relation [16]

$$
\varepsilon_{1}=C_{x} d / \varepsilon_{0} A,
$$

where $C_{x}$ is the capacitance of the sample, $A$ is the cross-sectional area of the parallel surfaces of the sample and $\varepsilon_{0}$ is the permittivity of the free space. The dielectric loss $\varepsilon_{2}$ (imaginary part of the dielectric constant) was calculated using the relation [17]

$$
\varepsilon_{2}=\varepsilon_{1} \tan \delta,
$$


where $\delta=90^{\circ}-\phi ; \phi$ is the phase angle. The ac conductivity $\sigma_{\mathrm{ac}}(\omega)$ is determined by the relation [17]

$$
\sigma_{\mathrm{ac}}(\omega)=\sigma_{\mathrm{tot}}(\omega)-\sigma_{\mathrm{dc}},
$$

where $\sigma_{\text {tot }}(\omega)$ is the total conductivity which is calculated from the relation

$$
\sigma_{\mathrm{tot}}(\omega)=\frac{d}{A} \frac{1}{z}
$$

where $Z$ is the total impedance of the sample.

\section{Results and discussion}

\subsection{Frequency and temperature dependence}

The frequency and temperature dependence of the ac conductivity $\sigma_{\mathrm{ac}}(\omega)$, the dielectric constant $\varepsilon_{1}$, and the dielectric loss $\varepsilon_{2}$ were studied for $\operatorname{In}_{2} \mathrm{Te}_{3}$ thin films of different thicknesses $170-300 \mathrm{~nm}$, in the frequency range $100 \mathrm{~Hz}-100 \mathrm{kHz}$ and in the temperature range $303-373 \mathrm{~K}$ to understand the conduction process in the $\operatorname{In}_{2} \mathrm{Te}_{3}$ system.

The variation of the ac electrical conductivity with the frequency of $\operatorname{In}_{2} \mathrm{Te}_{3}$ thin film of thickness $170 \mathrm{~nm}$ as an example is shown in Fig. 1 for several fixed temperatures. It is clear from the figure that $\sigma_{\mathrm{ac}}(\omega)$ increases linearly with increasing frequency according to the equation [17]

$$
\sigma_{\mathrm{ac}}(\omega)=A \omega^{s},
$$

where $s$ is the frequency exponent, and $\omega-$ the angular frequency. Values of $s$ were calculated from the slopes of the linear lines of this figure. It is clear also from the figure that $s$ is independent of the temperature in the investigated range, and it was found that $s=0.67$.

Figure 2 shows the relation between the ac conductivity $\sigma_{\mathrm{ac}}(\omega)$ and the temperature at different fixed frequencies of $\mathrm{In}_{2} \mathrm{Te}_{3}$ thin film of thickness $240 \mathrm{~nm}$. It is clear from this figure that $\sigma_{\mathrm{ac}}(\omega)$ increases linearly with the absolute temperature according to the well-known equation

$$
\sigma=\sigma_{0} \exp \left[-\Delta E_{\sigma}(\omega) / \mathrm{kT}\right],
$$

this suggested that the ac conductivity is a thermally activated process. The ac conduction activation energy $\Delta E_{\sigma}(\omega)$ is calculated for different frequencies from the slopes of these lines. There is no significant variation in the value of $\Delta E_{\sigma}(\omega)$ and this means that $\Delta E_{\sigma}(\omega)$ is frequency independent, its average calculated value was found to be $0.065 \mathrm{eV}$.

The QMT model [18] predicts a linear temperature dependence of $\sigma_{\mathrm{ac}}(\omega)$ and the exponent $s$ is almost equal to 0.8 and increases slightly or independent of the temperature which is given by [17]

$$
S=1-4 / \ln \left(1 / \omega \tau_{0}\right),
$$

where $\tau_{0}$ is a characteristic relaxation time. This means that our experimental results agree with the QMT model, so the frequency dependence of $\sigma_{\mathrm{ac}}(\omega)$ for $\mathrm{In}_{2} \mathrm{Te}_{3}$ can be explained in terms of QMT model. 


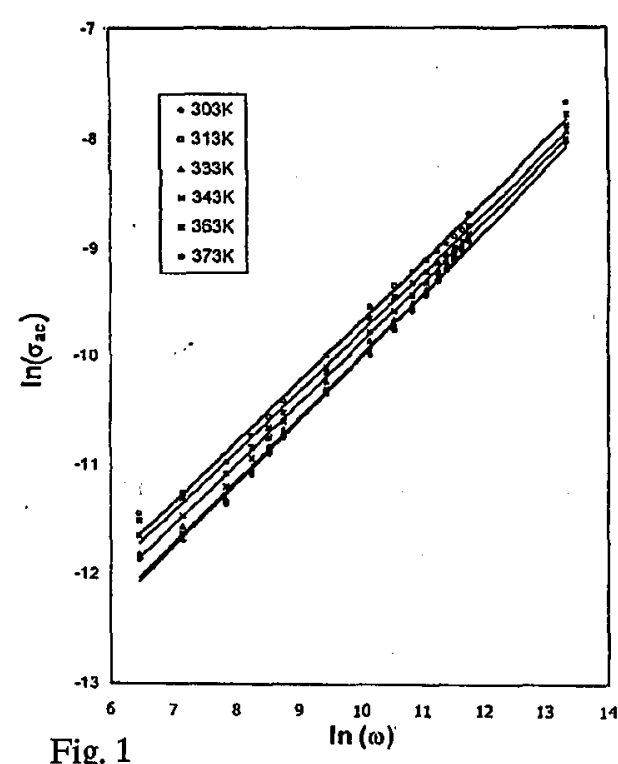

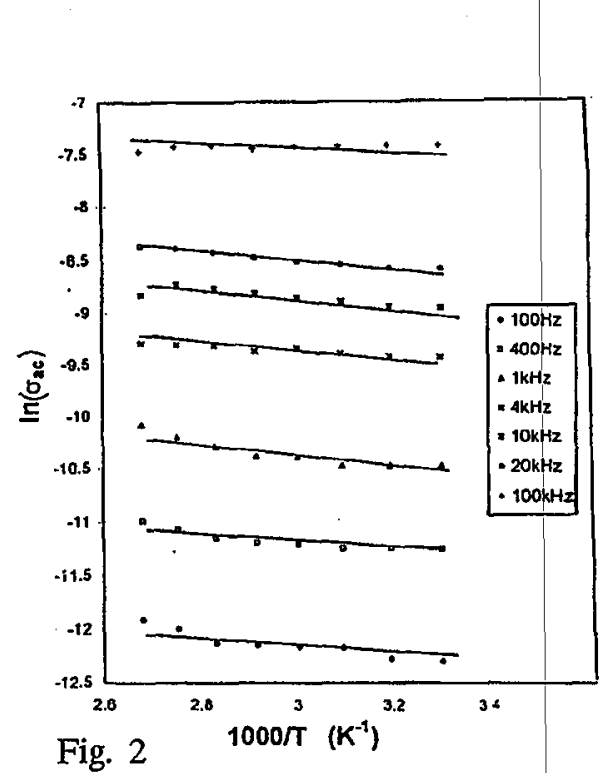

Fig. 2

Fig. 1. Frequency dependence of ac conductivity $\sigma_{\mathrm{ac}}(\omega)$ for the as-deposited $\mathrm{In}_{2} \mathrm{Te}_{3}$ film at different constant temperatures with thickness $170 \mathrm{~nm}$.

Fig. 2. The ac conductivity $\sigma_{\mathrm{ac}}(\omega)$ dependence on the temperature at different fixed frequencies of the as-deposited $\mathrm{In}_{2} \mathrm{Te}_{3}$ film of thickness $240 \mathrm{~nm}$.

According to QMT model, the ac conductivity $\sigma_{\mathrm{ac}}(\omega)$ is related to the density of states $N\left(E_{\mathrm{F}}\right)$ at the Fermi level by the following equation [19]:

$$
\left.\sigma_{\mathrm{ac}}(\omega)=(\pi / 3)\left[N\left(E_{\mathrm{F}}\right)\right]^{2} k T e^{2} \alpha^{-5} \ln \left(\nu_{\mathrm{ph}} / \omega\right)\right]^{4},
$$

where $T$ is the temperature, $N\left(E_{\mathrm{F}}\right)$ - the density of defect states at the Fermi energy $E_{\mathrm{F}}, \nu_{\mathrm{ph}}$ is the phonon frequency and $\alpha^{-1}-$ the spatial extent of the localized wave function. By assuming $\nu_{\mathrm{ph}}=10^{12} \mathrm{~s}^{-1}$ and $\alpha^{-1}=10 \AA$ [9], the density of states is calculated. The calculated values of $N\left(E_{\mathrm{F}}\right)$ of $\operatorname{In}_{2} \mathrm{Te}_{3}$ thin film of thickness $170 \mathrm{~nm}$ as an example at different temperatures are listed in Table. It is clear from Table that $N\left(E_{\mathrm{F}}\right)$ has values in the order of $10^{19} \mathrm{eV}^{-1} \mathrm{~cm}^{-3}$ which decreases with the frequency and increases with the temperature.

The variation of the dielectric constant $\varepsilon_{1}$ with the frequency at different constant temperatures for $\mathrm{In}_{2} \mathrm{Te}_{3}$ film with thickness $220 \mathrm{~nm}$ as an example is shown in Fig. 3. The dielectric constant $\varepsilon_{1}$ decreases with increasing frequency. This decrease is more sharp at lower frequency and higher temperature and can be attributed to the fact that, at low frequencies, $\varepsilon_{1}$ for polar material is due to the contribution of multicomponents of polarizability [20,21] (electronic, ionic, orientational and interfacial polarizability). When the frequency increases, the dipoles will no longer be able to rotate sufficiently rapidly, so $\varepsilon_{1}$ begins to decrease approaching a constant value at high frequencies. This value is due to interfacial polarization and is typical of nonpolar material.

It is clear also from Fig. 3 that the dielectric constant $\varepsilon_{1}$ increases with the increase in temperature for all the investigated range of frequency. The increase in 
TABLE

The dependence of the density of states $N\left(E_{F}\right)$ on the frequency, working temperature and annealing temperature.

\begin{tabular}{l|c|c|c|c|c|c|c|c|c}
\hline \hline \multirow{2}{*}{$\begin{array}{c}\text { Frequency } \\
{[\mathrm{kHz}]}\end{array}$} & \multicolumn{8}{|c|}{$N\left(E_{\mathrm{F}}\right) \times 10^{19}\left[\mathrm{eV}^{-1} \mathrm{~cm}^{-3}\right]$} \\
\cline { 2 - 10 } & 303 & 313 & 333 & 343 & 363 & 373 & As-deposited & $423 \mathrm{~K}$ & $473 \mathrm{~K}$ \\
\cline { 2 - 10 } & 5.10 & 5.30 & 5.45 & 5.75 & 6.00 & 6.10 & 4.00 & 4.96 & 5.90 \\
0.1 & 3.95 & 4.03 & 4.10 & 4.20 & 4.28 & 4.42 & 3.90 & 4.90 & 5.63 \\
0.4 & 3.75 & 3.77 & 3.80 & 3.90 & 3.97 & 4.15 & 3.87 & 4.82 & 5.50 \\
1 & 3.27 & 3.30 & 3.32 & 3.43 & 3.60 & 3.72 & 3.70 & 4.60 & 5.06 \\
4 & 3.08 & 3.15 & 3.13 & 3.17 & 3.22 & 3.35 & 3.45 & 4.20 & 4.63 \\
10 & 2.88 & 2.91 & 2.95 & 3.00 & 3.04 & 3.10 & 3.30 & 4.03 & 4.50 \\
20 & 2.62 & 2.78 & 2.70 & 2.75 & 2.8 & 2.92 & 3.20 & 3.70 & 4.00 \\
100 & & &
\end{tabular}

${ }^{*} N\left(E_{\mathrm{F}}\right)$ of annealed films was calculated at working temperature of $303 \mathrm{~K}$.

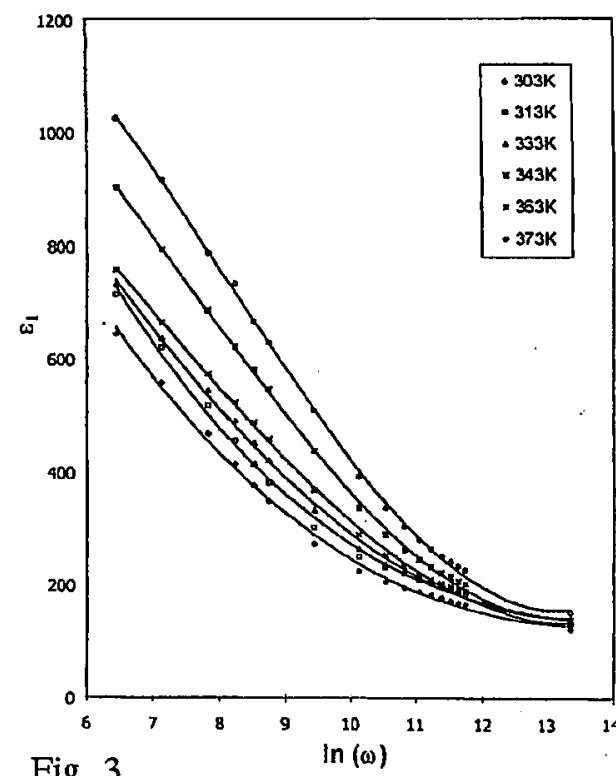

Fig. 3

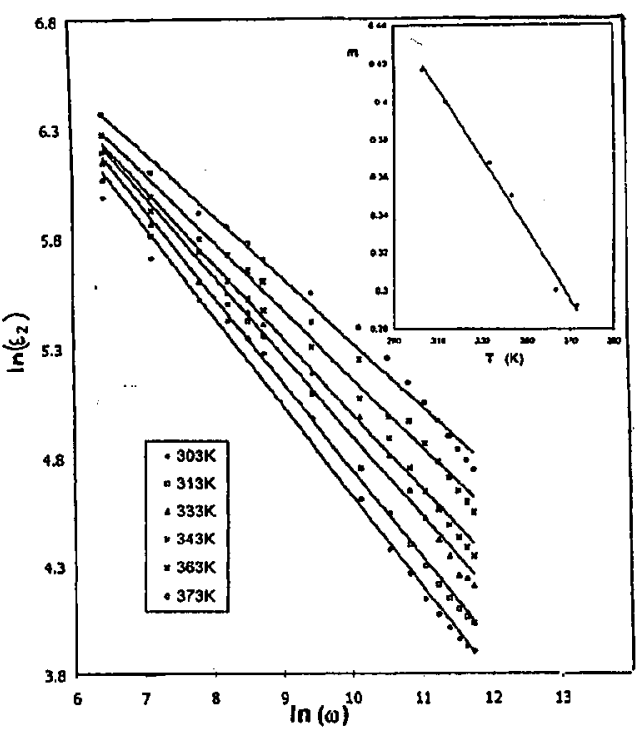

Fig. 4

Fig. 3. The dielectric constant $\varepsilon_{1}$ dependence on the frequency at different constant temperatures for the as-deposited $\operatorname{In}_{2} \mathrm{Te}_{3}$ film of thickness $220 \mathrm{~nm}$.

Fig. 4. The dependence $\ln \varepsilon_{2}$ vs. $\ln \omega$ for the as-deposited $\operatorname{In}_{2} \mathrm{Te}_{3}$ film of thickness $220 \mathrm{~nm}$ at different constant temperatures; the inset shows the temperature dependence of the calculated values of $m$ for the same film.

$\varepsilon_{1}$ with the temperature is more clear at lower frequencies and can be attributed to the fact that the dipoles in polar material cannot orient themselves at low 
temperatures and the orientation of dipoles is facilitated when the temperature is raised which increases the value of the dielectric constant $\varepsilon_{1}$.

The variation in the dielectric loss $\varepsilon_{2}$ as a function of temperature and frequency is similar to the results obtained for the dielectric constant $\varepsilon_{1}$, but the decrease in $\varepsilon_{2}$ with the frequency is higher at low temperature. This is clear from Fig. 4 which represents the relation between the dielectric loss $\varepsilon_{2}$ and the frequency at different constant temperatures as $\ln \left(\varepsilon_{2}\right)$ vs. $\ln (\omega)$ for a thin film with thickness $220 \mathrm{~nm}$ as an example according to the relation [22]

$$
\varepsilon_{2}=A \omega^{m}
$$

where $A$ is a constant. The power $m$ was calculated from the negative slopes of the obtained straight lines of Fig. 4 for different temperatures and is represented as a function of temperature in the inset of Fig. 4. It is shown that $m$ decreases linearly with the increase in temperature.

It is also clear from Fig. 4 that the dielectric loss $\varepsilon_{2}$ increases with temperature. This increase is more clear at higher frequencies and can be explained by Stevels [23] who divided the relaxation phenomena into three parts: conduction losses, dipolar losses and vibrational losses. At low temperatures, conduction losses have minimum value since it is proportional to $\sigma / \omega$ and $\sigma$ increases with increasing temperature, so the conduction losses increase which increases the value of dielectric loss $\varepsilon_{2}$.

\subsection{Effect of annealing}

To illustrate the effect of annealing on the ac electrical conductivity and the dielectric constants $\varepsilon_{1}, \varepsilon_{2}$ of $\operatorname{In}_{2} \mathrm{Te}_{3}$ thin films, several films of $\operatorname{In}_{2} \mathrm{Te}_{3}$ with different thicknesses were annealed at the temperature 373,423 , and $473 \mathrm{~K}$ for four hours. X-ray diffraction patterns indicate that $\mathrm{In}_{2} \mathrm{Te}_{3}$ thin films annealed at $423 \mathrm{~K}$ and then at $473 \mathrm{~K}$ have a polycrystalline structure and the degree of crystallinity increases by increasing the annealing temperature as shown in Fig. 5. During the process of annealing, the atomic orientation to the planes of the as-deposited $\mathrm{In}_{2} \mathrm{Te}_{3}$ becomes easier and besides, more reflecting planes appear indicating polycrystalline growth associating the full crystallinity of the investigated films. Results of careful analysis for this pattern together with JCPDS card no. 33-1488 indicates polycrystalline nature of $\beta-\operatorname{In}_{2} \mathrm{Te}_{3}$. The composition of the annealed investigated films was checked by energy dispersive X-ray (EDX) spectroscopy and it was found to be the same as that of the as-deposited films.

For annealed films of different thicknesses, no distinguishable changes in the ac electrical conductivity, dielectric constant $\varepsilon_{1}$, and dielectric loss $\varepsilon_{2}$ were observed for films annealed at $373 \mathrm{~K}$ in comparison with the as-deposited films. However, the films annealed at 423 and $473 \mathrm{~K}$ show significant variation. The variation of ac conductivity with the annealing temperature is shown in Figs. 6 and 7 .

Figure 6 shows the frequency dependence of $\sigma_{\mathrm{ac}}(\omega)$ for film annealed at $423 \mathrm{~K}$, then at $473 \mathrm{~K}(t=300 \mathrm{~nm})$ as an example. It is clear from this figure that at any frequency, $\sigma_{\mathrm{ac}}(\omega)$ increases with increasing the annealing temperature and the same results are obtained for all measuring temperatures. The calculated 


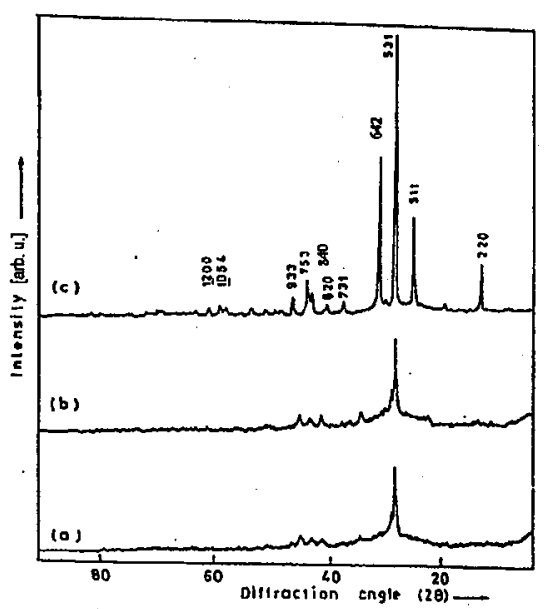

Fig. 5

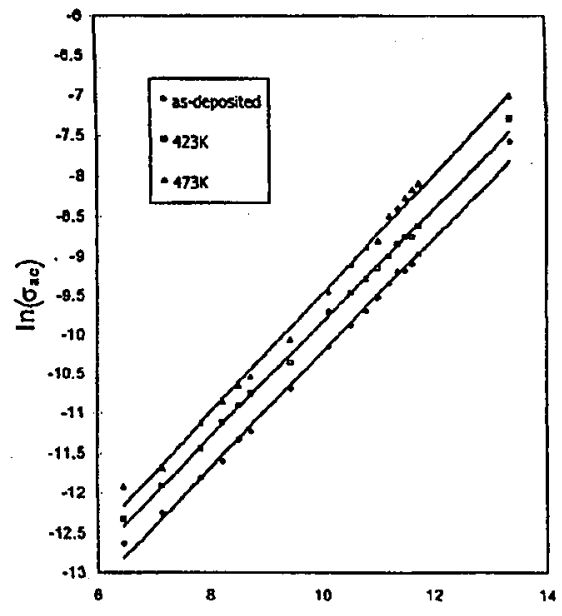

Fig. 6

$\ln (\omega)$

Fig. 5. X-ray diffraction pattern of $\operatorname{In}_{2} \mathrm{Te}_{3}$ thin films (a) as-deposited, $(b)$ annealed at $423 \mathrm{~K},(c)$ annealed at $473 \mathrm{~K}$.

Fig. 6. Frequency dependence of ac conductivity $\sigma_{\mathrm{ac}}(\omega)$ measured at room temperature for $\mathrm{In}_{2} \mathrm{Te}_{3}$ thin film of thickness $300 \mathrm{~nm}$ annealed at different temperatures.

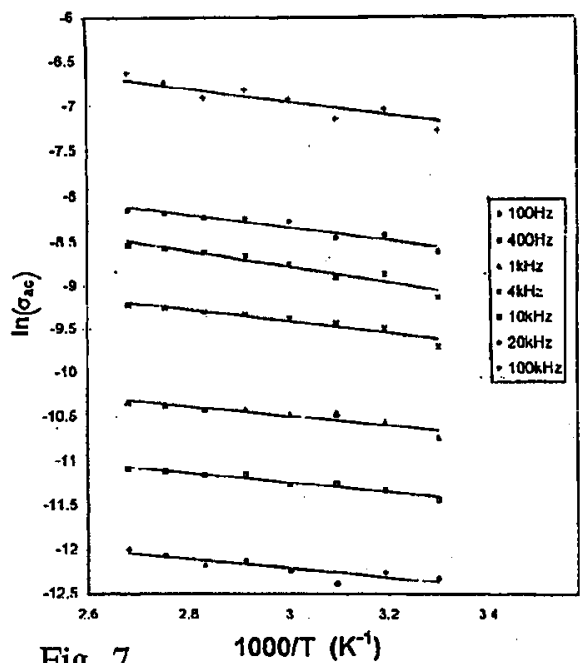

Fig. 7

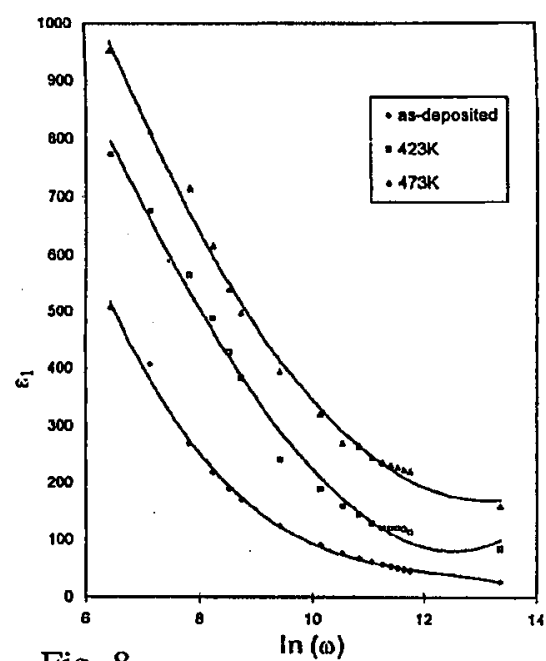

Fig. 8

Fig. 7. Temperature dependence of the ac conductivity for $\operatorname{In}_{2} \mathrm{Te}_{3}$ film of thickness $300 \mathrm{~nm}$ annealed at $423 \mathrm{~K}$ at different fixed frequencies.

Fig. 8. Frequency dependence of the dielectric constant $\varepsilon_{1}$ measured at room temperature for $\mathrm{In}_{2} \mathrm{Te}_{3}$ film of thickness $300 \mathrm{~nm}$ annealed at different temperatures.

values of $s$ show that it is independent of the thickness, measuring and annealing temperature, and have the same values as that of the as-deposited films. 
The temperature dependence of $\sigma_{\mathrm{ac}}(\omega)$ for $\operatorname{In}_{2} \mathrm{Te}_{3}$ film with thickness $300 \mathrm{~nm}$ annealed at $423 \mathrm{~K}$ is shown in Fig. 7 at different constant frequencies. It is clear from this figure that $\sigma_{\mathrm{ac}}(\mathrm{w})$ for the annealed films increase linearly with increasing temperature according to Eq. (8). The calculated values of $\Delta E_{\sigma}(\omega)$ are found to be independent of the thickness and frequency in the investigated range. Its average value is found to be $0.054 \mathrm{eV}$ and $0.043 \mathrm{eV}$ for films annealed at 423 and $473 \mathrm{~K}$ respectively and this means that $\Delta E_{\sigma}(\omega)$ decreases with increasing the annealing temperature and this is due to the increase in the degree of crystallinity to a more ordered state by annealing. It is also clear from Fig. 8 that $\sigma_{\mathrm{ac}}(\omega)$ increases with the increase in frequency.

The calculated values of $N\left(E_{\mathrm{F}}\right)$ for films annealed at 423 and $473 \mathrm{~K}$ are listed also in Table. It is clear that $N\left(E_{\mathrm{F}}\right)$ increase with the annealing temperature at any frequency.

The change of the dielectric constant $\varepsilon_{1}$ and the dielectric loss $\varepsilon_{2}$ with the annealing temperature is shown in Figs. 8 and 9 which show the frequency dependence of the room temperature $\varepsilon_{1}$ and $\varepsilon_{2}$ for $\operatorname{In}_{2} \mathrm{Te}_{3}$ films with thicknesses 300 and $240 \mathrm{~nm}$ respectively annealed at different temperatures. It is clear from these figures that $\varepsilon_{1}$ and $\varepsilon_{2}$ increase with annealing temperature at any frequency and decrease as the frequency increases. This is also attributed to the increase in the degree of crystallinity.

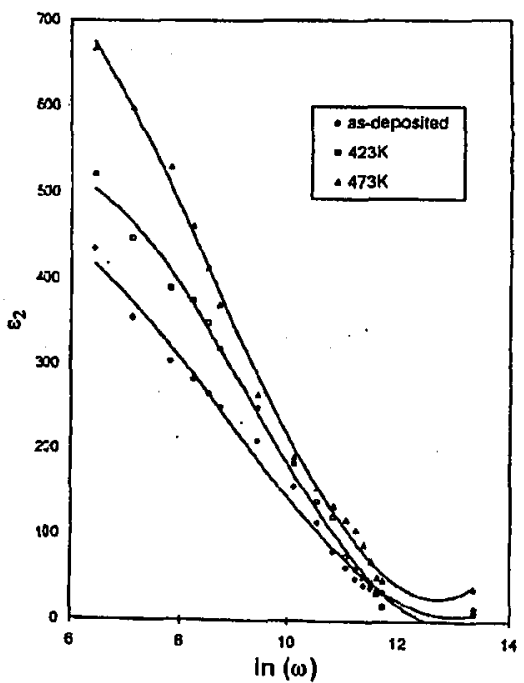

Fig. 9. Frequency dependence of the dielectric loss $\varepsilon_{2}$ measured at room temperature for $\mathrm{In}_{2} \mathrm{Te}_{3}$ film of thickness $240 \mathrm{~nm}$ annealed at different temperatures.

In conclusion, the data of ac conductivity and dielectric properties for as-deposited and annealed $\mathrm{In}_{2} \mathrm{Te}_{3}$ films can be explained on the basis of the QMT models which assumed that carrier motion occurs through quantum-mechanical tunneling between localized (defect) states near the Fermi level. 


\section{References}

[1] K. Kambas, J. Spyridelis, Mater. Res Bull. 13, 653 (1978).

[2] C. Julien, M. Eddrief, K. Kambas, M. Balkanski, Thin Solid Films 137, 27 (1986).

[3] A.I. Zaslavskii, N.F. Kartenko, Z.A. Karachentseva, Sov. Phys.-Solid State 13, 2152 (1972).

[4] A.I. Zaslavskii, V.M. Sergeeva, Sov. Phys.-Solid State 2, 2556 (1961).

[5] G.L. Bleris, T. Karakostas, J. Stoemenos, N.A. Economou, Phys. Status Solidi A 34, 243 (1976).

[6] T. Karakostas, N.A. Economou, Phys. Status Solidi A 31, 89 (1975).

[7] G.L. Bleris, T. Karakostas, N.A. Economou, R. Deridder, Phys. Status Solidi A 50, 579 (1978).

[8] V.M. Kosevich, A.A. Sokal, A.D. Barrinok, Sov. Phys.-Crystallogr. 29, 212 (1984).

[9] A. Zahab, M. Abd-Lefdil, M. Cadene, Phys. Status Solidi A 117, K 103 (1990).

[10] A. Zahab, M. Abd-Lefdil, M. Cadene, Phys. Status Solidi A 115, 491 (1989).

[11] M.A. Afif, N.A. Hegab, A.E. Bekheet, Vacuum 47, 265 (1996).

[12] N.A. Hegab, A.E. Bekheet, M.A. Afifi, A.E. El-Shazly, Appl. Phys. A 66, 235 (1998).

[13] N.A. Hegab, M.A. Afifi, A.E. El-Shazly, A.E. Bekheet, J. Mater. Sci. 33, 2441 (1998).

[14] M. Pollak, T.H. Geballe, Phys. Rev. B 22, 1742 (1961).

[15] D.P. Singh, K.D. Kundra, J. Mater. Sci. Lett. 8, 524 (1989).

[16] A.K. Shavna, K.L. Bahata, J. Non.-Cryst. Solids 109, 95 (1989).

[17] A. Ghosh, Phys. Rev. B 42, 5665 (1990).

[18] A. Ghosh, Phys. Rev. B. 41, 1479 (1990).

[19] I.G. Austin, N.F. Mott, Adv. Phys. 18, 41 (1969).

[20] B. Tareev, Physics of Dielectric Materials, Mir, Moscow 1975.

[21] A.A. Zaky, R. Hawley, Dielectric Solids, Routledge \& Kegan Paul Ltd., London 1970, p. 16.

[22] J.S. Giuntini, J.V. Zanchetta, J. Non.-Cryst. Solids 45, 57 (1981).

[23] J.M. Stevels, Handbuch der Physik, Ed. S. Flügge, Vol. XX, Springer, Berlin 1957, p. 350 . 